\title{
Les chemins de fer d'intérêt local de l'Hérault dans le Biterrois de 1850 à 1950
}

Raoul Balso

\section{(2) OpenEdition}

\section{Journals}

Édition électronique

URL : https://journals.openedition.org/rhcf/2046

DOI : 10.4000/rhcf.2046

\section{Éditeur}

Rails \& histoire

\section{Édition imprimée}

Date de publication : 2 mai 2002

Pagination : 181-189

ISBN : 00996-9403

ISSN : 0996-9403

\section{Référence électronique}

Raoul Balso, «Les chemins de fer d'intérêt local de l'Hérault dans le Biterrois de 1850 à 1950 », Revue d'histoire des chemins de fer [En ligne], 24-25 | 2002, mis en ligne le 13 avril 2015, consulté le 22 avril 2022. URL : http://journals.openedition.org/rhcf/2046; DOI : https://doi.org/10.4000/rhcf.2046 


\section{Les chemins de fer $d^{\prime}$ intérêt local de l'Hérault dans le Biterrois de 1850 à 1950}

Sitôt après le vote de la loi sur les chemins de fer d'intérêt local, le 12 juillet 1865, le conseil général de l'Hérault décide la création d’un réseau départemental qui sera construit par le département avec le concours financier des communes et une subvention de l'État.

Ce réseau à voie unique et à écartement normal (1,44 m), d'une longueur totale de $200 \mathrm{~km}$, comportera 8 lignes. L'enquête préliminaire est menée dès juin 1866 et les Mémoires descriptifs de M. Fenouil, l'agent voyer en chef, estiment le prix de revient moyen de chaque ligne. Dans le Biterrois, les prix atteignent $54200 \mathrm{~F}$ pour la ligne de Béziers à Pézenas et $88000 \mathrm{~F}$ pour la ligne de Béziers à Saint-Chinian.

En septembre 1866 l'appel d'offres est lancé. L'ingénieur conseil Henri Joret, de Paris, candidat soutenu par l'ingénieur en chef des Ponts et Chaussées Bazaine (le frère du maréchal) est sélectionné. Par décret impérial du 14 août 1867 signé au camp de Châlons, la concession du nouveau réseau est accordée à la Compagnie des chemins de fer de l'Hérault. Cette société anonyme au capital social de 5 millions de francs -10000 obligations de $500 \mathrm{~F}$ remboursables en 80 ans - a des bureaux au 5, rue Maguelonne, à Montpellier mais reste domiciliée au 80, rue Taitbout, à Paris.

Les travaux commencent par la ligne de Montpellier à Palavas, qui est inaugurée le 9 mai 1872. Dans le Biterrois, la construction des lignes s'échelonne sur plusieurs années : la section Béziers-Pézenas s'achève en 1874 mais l'ensemble de la ligne Béziers-Saint-Chinian n'est en service que le 23 novembre 1887.

Mon exposé comprend trois volets correspondant aux trois grands thèmes du colloque : la conquête des campagnes ; l'aménagement du territoire ; histoire et patrimoine. Mais, d'abord, il m'a paru nécessaire de situer brièvement le contexte économique exceptionnel dans lequel ont été créés et ont fonctionné ces chemins de fer d'intérêt local. 


\section{Essor du Biterrois dans la seconde moitié du XIX ${ }^{\mathrm{e}}$ siècle}

Dans la seconde moitié du XIX ${ }^{\mathrm{e}}$ siècle et au début du XX' siècle, Béziers a connu une remarquable prospérité. Sa population est passée de 20000 habitants en 1850 à 35000 habitants en 1870, 50000 habitants en 1900 et 70000 habitants en 1939. Une étude de ses négociants en vin présentée récemment à un colloque de l'Université Paul-Valéry à Montpellier m'a donné l'occasion d'en éclairer l'origine ${ }^{1}$.

\section{Viticulture}

Depuis 1842, les mercuriales des marchés de Béziers et de Pézenas fixent les prix des vins et des eaux-de-vie dans tout le royaume. La production locale atteint déjà 2,1 millions d'hectolitres de vin dont 400000 sont consommés sur place, 400000 exportés et 1,3 millions brûlé puis exporté sous forme de trois-six ${ }^{2}$.

Tous les vendredis, sur les allées Paul-Riquet de Béziers, des négociants de Montpellier, Bordeaux, Lyon, Marseille et Paris se rencontrent et arrêtent les prix. C'est pourquoi, lors de la crise viticole qui suit la Révolution de février 1848, les viticulteurs de la région, réunis à Narbonne, déclarent que « Béziers doit marcher en tête de la croisade entreprise par les baissiers et coordonner l'action des quatre départements intéressés : l'Aude, les Pyrénées-Orientales, le Gard et l'Hérault »³.

Le dimanche 3 avril 1851, une assemblée de 100 propriétaires réunis à Béziers élit une commission chargée de diriger l'action viticole du Midi : elle est présidée par Lognos, le maire de Béziers. Le dispositif qui va assurer la prépondérance du Biterrois pour plus d'un siècle est en place.

\section{Transport}

A la même époque, la construction de voies ferrées redonne sa vitalité au carrefour économique biterrois. En 1857, la puissante Compagnie du Midi, des frères Pereire, met en service la section Béziers-Sète qui assure la jonction avec le P.L.M. et réalise la liaison Océan-Méditerranée prévue par la loi du 11 juin 1842.

En 1865, la Compagnie du Midi rachète la ligne minière de Béziers à Graissessac et la prolonge de Bédarieux vers le Nord pour avoir un accès à Paris indépendant de ses rivales, le P.L.M. et le P.O. Atteignant Tournemire en 1872, Millau en 1874, Saint-Flour en 1884 et Neussargues en 1888, elle fait de Béziers un nœud ferroviaire important.

Le géographe Marcel Blanchard a pu écrire que : « La capitale du vin ne s'appuie pas seulement sur la prospérité du vignoble mais aussi sur la disposition des voies ferrées qui convergent vers elle ${ }^{4}$. » De fait, 
les chemins de fer de l'Hérault n'ont plus à régler que des problèmes d'intérêt local.

\section{La conquête des campagnes}

Au départ de Béziers, la conquête des campagnes s'est faite vers l'Est en direction de Pézenas et vers l'Ouest en direction de Saint-Chinian à travers deux régions très différentes.

\section{La ligne Béziers-Pézenas}

La construction de la ligne de Béziers à Pézenas (27 km), joignant la vallée de l'Orb à celle de l'Hérault, a été une revanche pour toutes les communes qui, dans les années 1840, avaient perdu « la guerre des tracés ». Les études de factibilité menées alors par les ingénieurs des Ponts et Chaussées (rapport Blondat de 1843-1844) avaient conclu en faveur du tracé littoral de Béziers à Sète via Agde : prix de revient moins élevé, population desservie plus nombreuse.

La nouvelle ligne, à travers une plaine légèrement vallonnée qu'arrosent la Tongue, la Peyne et le Libron, dessert les six communes de Boujan, Bassan, Servian, Valros, Tourbes et Pézenas. Inaugurée en 1874, elle témoigne d'une volonté de mise en valeur du territoire. Comme le remarque l'agent voyer Fenouil, ce chemin de fer s'inscrit dans la ligne de la loi du 21 mai 1836 sur les chemins vicinaux ${ }^{5}$.

\section{La ligne Béziers-Saint-Chinian}

La construction de la ligne de Béziers à Saint-Chinian (44 km) a une tout autre histoire. Remontant la vallée de l'Orb, la ligne s'enfonce dans les Soubergues, c'est-à-dire dans les avant-monts du massif de l'Espinouse.

Dès l'enquête préliminaire de 1865, le conseil général demande «que ce chemin de fer soit prolongé jusqu'à Saint-Pons pour faciliter l'échange des produits de la plaine avec ceux de la partie montagneuse de l'arrondissement de Saint-Pons ». Le manque d'intérêt des élus de Saint-Pons, axés sur leur industrie textile, fit abandonner le projet d'une section Saint-Chinian-Saint-Pons dont le coût s'avérait très élevé avec un prix moyen du kilomètre estimé à près de 3 millions de francs.

La ligne desservit les huit communes de Maraussan, Maureilhan, Lignan, Cazouls-les-Béziers, Riols, Cessenon, Pierrerue et Saint-Chinian. La gare de Saint-Chinian, installée au pied du château des anciens évêques, resta le terminus occidental du réseau et les plateaux couverts de garrigue menant vers la vallée du Jaur restèrent sans chemin de fer. Avec le grand pont métallique de Tabarka et les gorges de Réals, la 
section Béziers-Saint-Chinian avait déjà posé de gros problèmes techniques aux ingénieurs des Ponts et Chaussées et montré les limites de la rentabilité des investissements ferroviaires.

\section{L'aménagement du territoire}

Tout le long du réseau, les exemples d'aménagement du territoire sont nombreux. J'évoquerai le plus marquant, celui de la transformation de la zone nord de la ville de Béziers, pour lequel les archives municipales sont un bon document.

En 1873, la gare de la section Pézenas-Béziers vint tout naturellement se situer le long de la route nationale $n^{\circ} 9$, arrivant de Pézenas. À l'entrée de la ville, la commune octroya à la compagnie 3,59 ha dans une zone encore non urbanisée. Le bâtiment voyageurs, en longueur, fut installé côté route et une cour d'accès fut aménagée. La cour marchandises s'ouvrit de l'autre côté des voies, vers la place qui servait de marché aux bestiaux. Le dépôt et les ateliers s'établirent au pied du Pech-du-Rouat qui distribuait l'eau des bassins réservoirs.

En 1876, lorsque la section Saint-Chinian-Béziers entra en service, d'importants travaux de terrassement furent nécessaires pour que la route nationale $n^{\circ} 9$ enjambe les voies : le trafic était trop important, surtout sur la route, pour que la solution de passages à niveau soit retenue.

Cette gare du Nord vint faire contrepoids à la gare du Midi et contribua au développement d'un nouveau quartier ainsi qu'en témoigne un rapport de l'agent voyer adressé au préfet, le 28 novembre 1878 : «Aujourd'hui, par suite de l'agrandissement de la ville dans le quartier de la gare du Nord, par le transfert du marché aux bestiaux et par la construction de la caserne de cavalerie dans l'ancien champ de Mars, les abords du chemin de fer d'intérêt local demandent à être dégagés. La gare n'est plus dans un faubourg reculé de la ville : elle est au contraire entourée de rues ou de chemins qu'il faut ramener à des pentes normales pour mettre en communication le marché aux bestiaux avec l'abattoir et la nouvelle caserne avec la route nationale $n^{\circ}$ 9, artère principale de la ville de Béziers ${ }^{6} \ldots$ » En 1884, après que la ville a souscrit un emprunt de $966000 \mathrm{~F}$, s'achève la construction du grand mur de soutènement de $20 \mathrm{~m}$ de haut qui domine toujours la rue de l'Hérault.

En 1902, une des lignes du nouveau tramway électrique assure une liaison avec le centre de la ville, complétant des services de roulage insuffisants et, très rapidement, au milieu de vignobles transformés en 
lotissements, un quartier se développe dont la population passe de 363 habitants à 3452 habitants (dénombrement de 1911).

\section{Analyse du réseau}

Dans la série $S$ des archives départementales de l'Hérault, trois types de documents permettent de reconstituer les caractéristiques de ce réseau d'intérêt local : les notices techniques éditées par les Ponts et Chaussées ; les recueils de tarifs voyageurs et marchandises ; les comptes rendus annuels d'exploitation qui traitent du matériel roulant, du service des trains et de la gestion du personnel.

\section{Caractéristiques techniques de la voie}

Sous le Second Empire, le chemin de fer, malgré le développement rapide des réseaux, est encore une technique nouvelle qui étonne les populations rurales. L'agent voyer Péréal juge nécessaire de préciser: " Bien des personnes ont cru et croient encore que nous allons établir des chemins de fer à traction de chevaux. C'est une erreur qu'il importe de dissiper. Les voies du département de l'Hérault seront, comme toutes les autres voies ferrées, desservies par des locomotives ${ }^{7}$. »

Pour la construction des lignes ferroviaires qui diffère sensiblement de celle des routes, la direction des Ponts et Chaussées édite des notices destinées aux ingénieurs : « Notices pour l'étude et la construction des chemins de fer en conformité avec la circulaire ministérielle du 28 juin 1879. » Y sont rappelées les règles relatives à la voie, aux traverses et au ballast, aux talus, ponts et aux ponceaux, aux passages à niveau (barrières roulantes ou croix de Saint-André).

Une autre notice définit les divers types de stations et de maisons de garde annexés à la circulaire de 1880 . De nombreux ingénieurs présentent les études qu'ils ont faites pour rendre la construction et l'exploitation des chemins de fer d'intérêt local moins coûteuses : ainsi une brochure de J. Larmanjat ${ }^{8}$.

\section{Matériel roulant - Service des trains}

Un état de 1879 nous apprend que ce réseau secondaire dispose de 14 locomotives sortant des ateliers Cail et Cie à Paris et pouvant rouler à $50 \mathrm{~km} / \mathrm{h}^{9}$. Le parc marchandises comporte 42 wagons plats, 10 wagons à houille, 10 wagons à bestiaux et 32 wagons à ballast.

Le parc voyageurs, relativement important, comprend 4 voitures de luxe, 26 voitures de $2^{\mathrm{e}}$ classe, 12 voitures mixtes ( $\left(^{\text {re }}\right.$ et $2^{\mathrm{e}}$ classe) et 8 voitures mixtes ( $2^{\mathrm{e}}$ classe et fourgon). Une note précise quelles doivent être les dimensions minima de la place affectée à chaque voyageur 
assis : largeur $42 \mathrm{~cm}$, profondeur $65 \mathrm{~cm}$, hauteur $150 \mathrm{~cm}$. Les voitures doivent être couvertes, garnies de banquettes avec dossiers, fermées avec des glaces et munies de rideaux.

Le service des trains comprenait des trains voyageurs mixtes, des trains marchandises et des trains marchandises mixtes. L'ensemble couvrait annuellement environ 360000 kilomètres à une vitesse moyenne de $24 \mathrm{~km} / \mathrm{h}$. Entre Béziers-Pézenas et Béziers-Saint-Chinian, le service voyageurs offrait quatre trains dans chaque sens. Tous étaient omnibus.

\section{Tarification}

Les tarifs voyageurs et marchandises publiés périodiquement se présentaient sous la forme de grandes plaquettes à couverture grise ${ }^{10}$. Les tarifs à grande vitesse comprenaient les tarifs voyageurs, avec les barèmes de prix, les tarifs de bagages, les tarifs de messagerie (denrées périssables, finances) et les tarifs d'animaux vivants (bœufs, porcs, moutons). Les tarifs à petite vitesse reprenaient toutes les autres marchandises, réparties en quatre classes.

Les tarifs spéciaux sont intéressants parce qu'ils indiquent les trafics les plus représentatifs : PV1 futailles vides ; PV2 marchandises expédiées par lots de $1000 \mathrm{~kg}$; PV3 objets de dimensions exceptionnelles ; PV4 emballages vides en retour ; PV7 foins en botte, fourrages ; PV8 alcools, eaux-de-vie, trois-six, vin... Le PV no 106 concernant les transports de vin en wagons foudre sur Paris et la région nord soulevait des problèmes avec les grandes compagnies, les voies des chemins de fer de l'Hérault n'acceptant que des charges de 11 tonnes à l'essieu.

La Compagnie des chemins de fer de l'Hérault n'a pas beaucoup de liberté en matière tarifaire. A son échelle, elle essaie cependant de pratiquer une politique commerciale : mise en route de trains spéciaux pour les fêtes locales (pose de la première pierre de la caserne Duguesclin le 19 mars 1876 ; " fêtes latines » de Montpellier en mai 1878) ou octroi des conditions spéciales pour le chargement ou le déchargement de marchandises en pleine voie, hors des stations.

\section{Personnel}

En 1880, l'ingénieur Bergeron, se basant sur des expériences allemandes et écossaises, affirmait dans sa « Notice sur les chemins de fer départementaux $»^{11} \mathrm{qu}^{\prime} \ll$ avec une petite compagnie et une direction locale et indépendante, un embranchement doit être toujours rémunérateur ». Les expériences françaises lui donnèrent tort. 
Les Chemins de fer de l'Hérault étaient une petite compagnie qui comptait moins de 300 personnes, à savoir 10 dirigeants, 121 agents mouvement (trafic), 83 agents traction et 84 agents de la voie, dont 4 femmes garde-barrières). Leur direction localisée à Montpellier était indépendante dans les limites accordées par les grandes compagnies encadrantes. Leur histoire n'est pourtant qu'un long déclin et, dans le Biterrois, ils n’ont laissé aucun patrimoine.

\section{Histoire et patrimoine}

\section{Du chemin de fer à l'automobile}

Après la guerre de 1914-1918, la situation financière de la compagnie, confrontée à une concurrence routière de plus en plus forte, devient difficile. Le 30 novembre 1928, le département décide de racheter toutes les lignes concédées moyennant le paiement de 50 annuités de $430000 \mathrm{~F}$, et confie le réseau à la Société générale des chemins de fer économiques par une convention de six ans, renouvelable. Parallèlement, il met sur pied un service régulier de transports automobiles, par une autre convention passée avec la Société des transports départementaux de l'Hérault, le 21 octobre 1927.

Le trafic de la ligne Montpellier-Béziers-Saint-Chinian continuant de baisser (1 372000 voyageurs en 1932, 757000 en 1933 et 593000 en 1934) une suspension du service est proposée le 6 septembre 1933 par le conseil municipal de Béziers. Un décret du 16 novembre 1939 déclasse toutes les lignes d'intérêt local du département mais, pour faire face à la pénurie d'essence, il est abrogé par le gouvernement de Vichy. Le trafic reprend : 1709000 voyageurs en 1944, 1811000 en 1945, avant que les transports automobiles l'emportent définitivement. Autobus et camions se multiplient tandis que les lignes ferment les unes après les autres.

\section{La disparition du réseau}

De 1954 à 1969, en quinze ans, les chemins de fer d'intérêt local disparaissent. La dépose des voies commence en mars 1957 sur la ligne Béziers-Pézenas et s'achève en janvier 1969 sur la ligne Béziers-SaintChinian. En même temps, les gares sont vendues aux enchères et déclassées par les communes. A Béziers, la gare du Nord est démolie dans le premier semestre de 1969 pour faire place à une ZAC.

Aujourd'hui, l'emplacement de l'ancienne gare est occupé par des services administratifs (poste centrale, bureaux des impôts, garages de l'Équipement) des résidences et des maisons de retraite groupées 
autour d'une esplanade, un parking pour le parc des sports proche et un jardin public. Le tracé des voies a été utilisé pour le passage de deux voies rapides permettant à la circulation routière d'éviter le centre ville.

Plus rien ne rappelle l'activité ferroviaire qui, pendant presque un siècle, a animé ces lieux.

\section{Notes}

1- Colloque «Les vignobles du Midi (XVI" - XXe siècles)», 16-17 mars 2001, Centre d'histoire moderne et contemporaine de l'Europe méditerranéenne, université de Montpellier III.

2- Creuzé deLesser, Statistiques du département de l'Hérault, Montpellier, 1847.

3- Le Journal de Bériers, 1851, nº 13.

4- Marcel Blanchard, Essais historiques sur les premiers chemins de fer du Midi languedocien et la vallée du Rhône, Montpellier, 1935.

5- Archives départementales de l'Hérault (ADH), série 5 S 422.

6- Archives municipales de Béziers (AMB), dossier gare du Nord.

7- $\mathrm{ADH}$, série $5 \mathrm{~S} 824 / 827$, fonds des Ponts et Chaussées.

8- Paris, $1872 ; A D H$, série $5 \mathrm{~S} 422$, Tracés.

9- $\mathrm{ADH}$, série $5 \mathrm{~S} 492$, Matériel roulant.

10- ADH, série 5 S 545, Tarifs des années 1886 et 1891.

11- ADH, sérié 5 S 423, Enquêtes. 


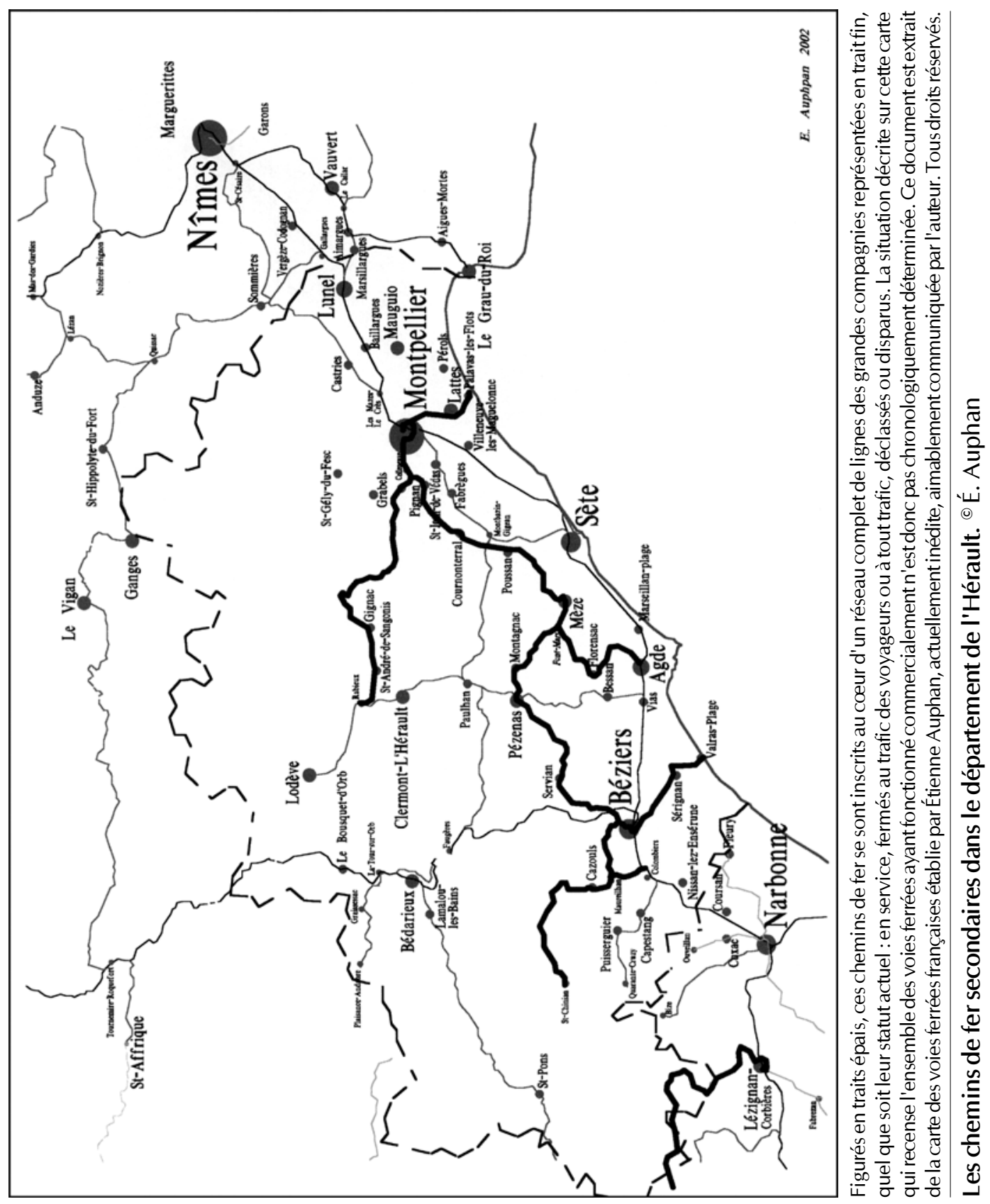

